\title{
ESTRATÉGIA PARA MIGRAÇÃO DOS SISTEMAS DE OTIMIZAÇÃO DE PROCESSO DOS ALTOS FORNOS DA ARCELORMITTAL TUBARÃO*
}

Bruno Valladão Fraga ${ }^{1}$

\section{Resumo}

Esse trabalho tem como objetivo apresentar a estratégia adotada para a migração dos sistemas de otimização de processo dos três Altos Fornos da ArcelorMittal Tubarão. O desenvolvimento dos novos sistemas é baseado no Plano Diretor de Tecnologia de Automação (PDTA) que definiu a arquitetura, tecnologias e elaborou o Framework como base das aplicações. Para a migração dos sistemas foi elaborada uma especificação detalhada de todos os módulos e funcionalidades existentes, permitindo dessa forma a criação de uma camada com as principais regras de negócios relativas ao processo para serem reutilizados em todos os Altos Fornos. Essa camada deu origem ao Framework dos Altos Fornos. Os resultados obtidos já podem ser vistos nos três sistemas migrados para a nova plataforma, com um alto desempenho e estabilidade, garantido dessa forma a segurança operacional objetivada.

Palavras-chave: Altos fornos; Migração; Atualização; Sistema; Otimização; Framework; Funcionalidades; Especificação; Desenvolvimento; Tecnologia; Arquitetura; Servidor; Plataforma; TI; TA.

\section{STRATEGY FOR MIGRATION OF BLAST FURNACES PROCESS OPTIMIZATION SYSTEM OF ARCELORMITTAL TUBARÃO}

\begin{abstract}
This work aims to present the strategy adopted for the migration of process optimization systems of the three blast furnaces at ArcelorMittal Tubarao. The development of the new systems was based on the Director of Automation Technology Plan (PDTA) that defined the architecture, technologies and developed the Framework based applications. For the systems migration a detailed specification of all the modules and features was prepared, thus allowing the creation of a layer with key business rules relating to the process for reuse in all Blast Furnaces. This layer gave birth to the Framework of Blast Furnaces. The results can already be seen in the three systems migrated to the new platform, with high performance and stability, guaranteed thus objectified operational safety.

Keywords: Blast furnaces; Migration; Update; System; Optimization; Framework; Functionalities; Specification; Development; Technology; Architecture; Server; Platform; TI; TA.
\end{abstract}

1 Engenheiro da Computação, Especialista de Automação, Gerência de Área de Engenharia de Automação, ArcelorMittal Tubarão, Serra, ES, Brasil. 


\section{INTRODUÇÃO}

Os sistemas de otimização de processo dos Altos Fornos da ArcelorMittal Tubarão desempenham um papel fundamental no monitoramento do processo, fornecendo informações essenciais para a estabilidade operacional da planta.

As aplicações existentes encontravam-se em fim de vida útil, tanto de hardware quanto de software, elas foram desenvolvidas utilizando um conjunto de ferramentas que já estão obsoletas e descontinuadas (Fortran, BGE, DMQ), a execução dos sistemas é feita por servidores Alpha com sistema operacional OpenVMS, também não suportada mais pelo fabricante.

Motivado por esses fatores decidiu-se realizar a migração dos sistemas para a nova plataforma definida pelo Plano Diretor de Tecnologia de Automação (PDTA), que definiu a arquitetura, ferramentas, tecnologias e elaborou o Framework para o desenvolvimento de todos os novos sistemas de otimização de processo.

Essas novas tecnologias utilizadas para o desenvolvimento dos novos sistemas já vem alinhadas com a realidade da convergência cada vez maior entre as áreas de $\mathrm{TI}$ e TA, sempre visando aproveitar as melhores competências e habilidades de cada lado, com o objetivo de garantir o bom funcionamento, segurança e estabilidade dos processos produtivos.

A migração iniciou-se na fase de especificação detalhada de todas as funcionalidades dos sistemas atuais, com base nessas informações optou-se por elaborar o Framework dos Altos Fornos, onde estariam as principais regras de negócio e modelos matemáticos comuns aos três fornos.

\section{MATERIAIS E MÉTODOS}

O projeto de migração foi dividido em 4 grandes etapas, sendo elas:

- Especificação

- Desenvolvimento

- Testes integrados

- Implantação

\subsection{Especificação}

A especificação das funcionalidades e modelagem de dados do sistema foi totalmente realizada pela equipe de Automação, para isso, foi adotado o esquema de especificação e desenvolvimento modularizados, com isso foi possível realizar diversas atividades em paralelo, resultando dessa forma em ganho de produtividade. Todas as especificações e modelagem foram feitas utilizando-se a linguagem UML e detalhadas de forma a permitir que a migração do sistema pudesse ser realizada sem a necessidade de acesso ao código atual do sistema. Para cada caso de uso identificado foi elaborado um diagrama de classes, sequência e de dados. 


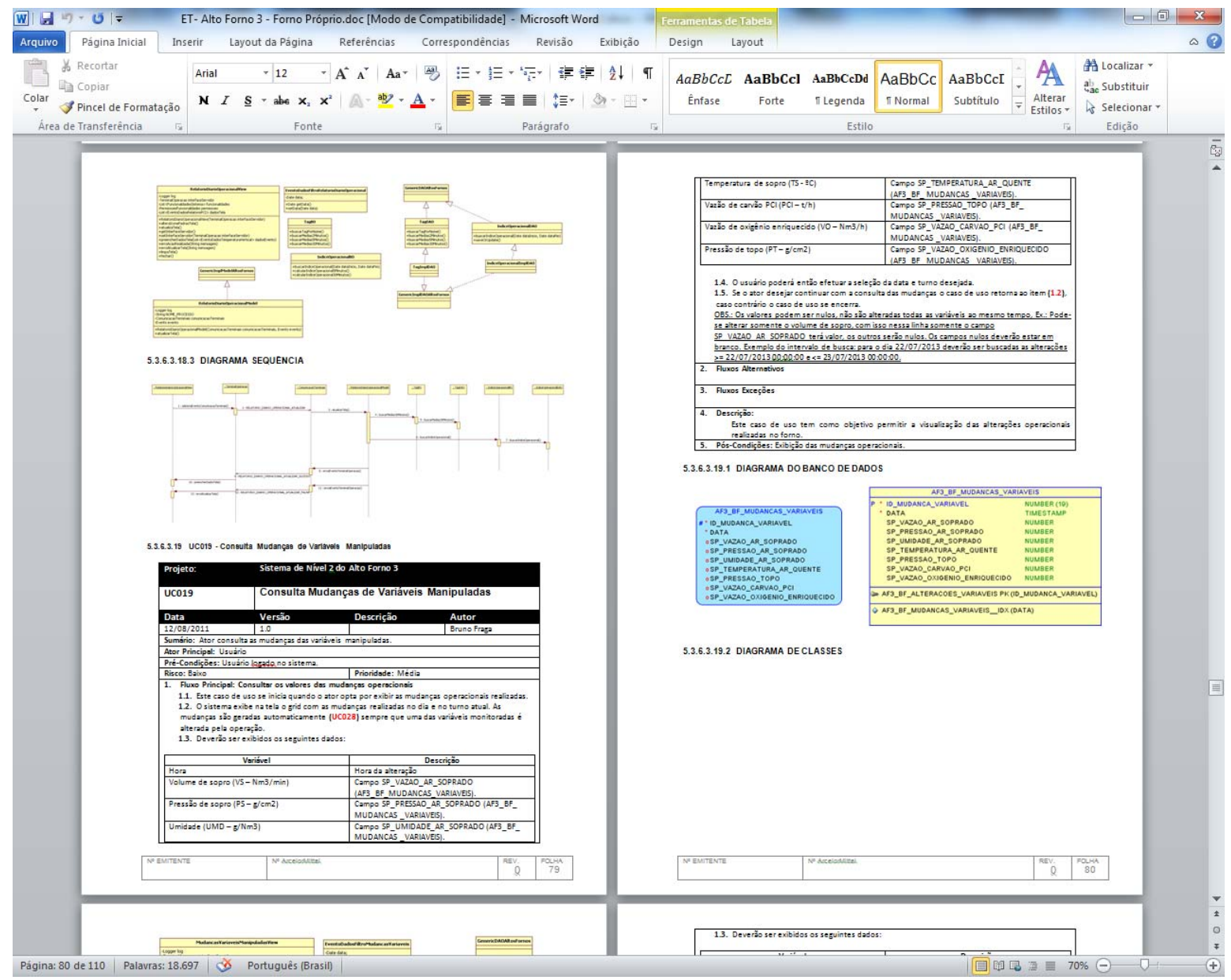

Figura 1. Especificação Funcional

\subsection{Desenvolvimento}

O desenvolvimento de cada módulo do sistema foi realizado por uma empresa parceira, tendo como base as especificações geradas no item anterior.

Essa etapa teve uma duração aproximada de 8 meses, e foram geradas aproximadamente 3000 classes para atender todo o sistema.

\subsection{Testes Integrados}

Após o desenvolvimento de cada módulo do sistema eram realizados os testes integrados com os outros módulos, com a comunicação entre sistemas e com o nível 1 , com isso foi possível garantir que ao final do último módulo a integração total do sistema.

\subsection{Implantação}

Após o fim de todos os testes e com o sistema validado iniciou-se a fase de implantação, que consistiu em uma semana de operação em paralelo do novo sistema, onde foi possível realizar a comparação de diversos dados operacionais e a correção dos problemas encontrados. Após a operação em paralelo o novo sistema foi colocado em produção com um acompanhamento $24 \times 7$ da equipe da Automação durante um período, de forma a garantir o seu perfeito funcionamento. 


\subsection{Framework}

A migração do sistema foi feita utilizando-se o Framework definido pelo PDTA, que é um conjunto de classes com objetivo de reutilização de um design, provendo um guia para uma solução de arquitetura em um domínio específico de software.

A utilização desse Framework trouxe diversos benefícios para o projeto, como:

- Redução do custo de desenvolvimento.

- Aumento do reuso (análise, design, código, etc.).

- Fez com que o desenvolvedor busque agregar funcionalidades ao invés de ficar reinventando a roda.

- Redução da quantidade de manutenções nos sistemas.

- Aumento da estabilidade dos sistemas e redução do número de defeitos devido ao uso em várias aplicações.

- Maior padronização dos sistemas.

- Aumento da compatibilidade dos sistemas.

- Disseminação do conhecimento dos especialistas sobre domínios de problemas de modo que o conhecimento não se perca com a saída do especialista.

A arquitetura desse Framework pode ser vista na Figura 2 abaixo. Cada módulo é responsável por uma determinada função do sistema, podendo a aplicação fazer uso ou não dela.

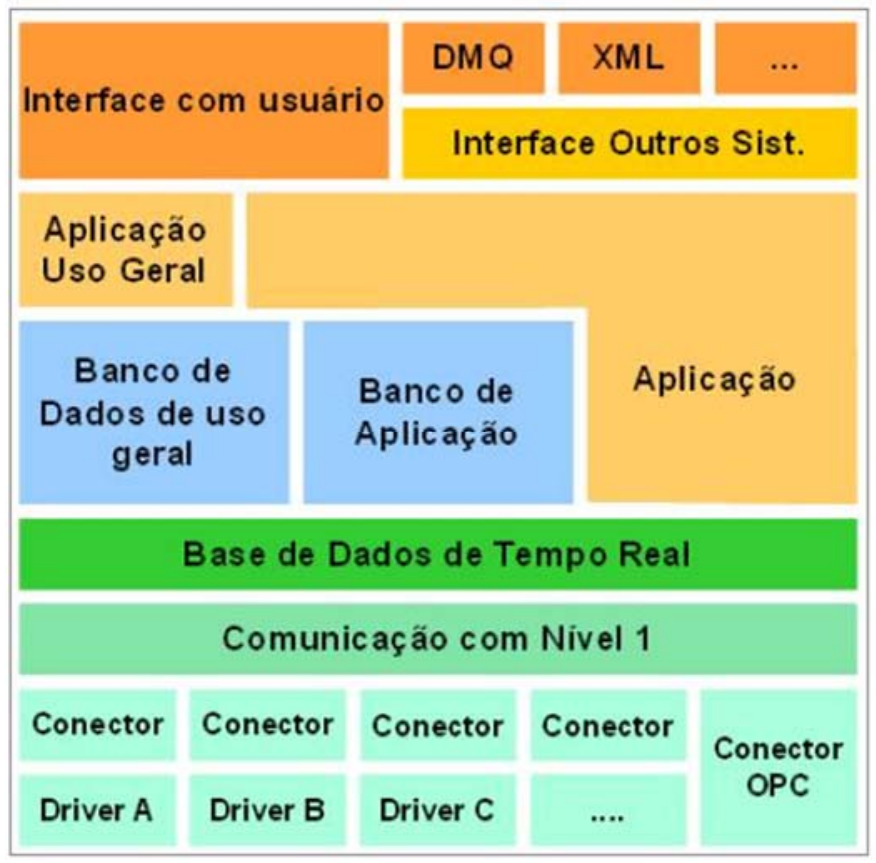

Figura 2. Framework da Automação

\subsection{Tecnologias Utilizadas}

Abaixo estão listadas as principais tecnologias empregadas no Framework e nos sistemas desenvolvidos:

- Sistema operacional: Windows Server 2008 R2 $\rightarrow$ O Windows Server 2008 R2 é um sistema operacional de servidor, produzido pela Microsoft. Foi liberado para fabricação em 22 de Julho de 2009, e lançado em 22 de Outubro de 2009. Ele se baseia no Windows NT 6.1, o mesmo sistema operacional básico usado no Windows 7 , orientado para o sistema cliente. É a 
primeira versão de sistema operacional exclusivamente 64 bits da Microsoft. O Windows Server 2008 R2 é uma atualização do Windows Server 2008 [1].

- Linguagem de programação: Java $1.7 \rightarrow$ Java é uma linguagem de programação interpretada orientada a objetos desenvolvida na década de 90 por uma equipe de programadores chefiada por James Gosling, na empresa Sun Microsystems. Diferente das linguagens de programação convencionais, que são compiladas para código nativo, a linguagem Java é compilada para um bytecode que é executado por uma máquina virtual. A linguagem de programação Java é a linguagem convencional da Plataforma Java, mas não é a sua única linguagem [1].

- IDE de desenvolvimento: NetBeans $\rightarrow$ O NetBeans IDE é um ambiente de desenvolvimento integrado () gratuito e de código aberto para desenvolvedores de software nas linguagens Java, C, C++, PHP, Groovy, Ruby, entre outras. O IDE é executado em muitas plataformas, como Windows, Linux, Solaris e MacOS. O NetBeans IDE oferece aos desenvolvedores ferramentas necessárias para criar aplicativos profissionais de desktop, empresariais, Web e móveis multiplataformas [1].

- Interface com usuário: Swing $\rightarrow$ Swing é um widget toolkit GUI (Interface de Usuário Gráfica) para uso com o Java. Ele é compatível com o Abstract Window Toolkit (AWT), mas trabalha de uma maneira totalmente diferente. A API Swing procura renderizar/desenhar por conta própria todos os componentes, ao invés de delegar essa tarefa ao sistema operacional, como a maioria das outras APIs de interface gráfica trabalham. Por ser uma API de mais alto nível, ou seja, mais abstração, menor aproximação das APls do sistema operacional, ela tem bem menos performance que outras APIs gráficas e consome mais memória RAM em geral. Porém, ela é bem mais completa, e os programas que usam Swing têm uma aparência muito parecida, independente do Sistema Operacional utilizado [1].

- Distribuição da aplicação cliente: Java Web Start $\rightarrow$ Em computação, Java Web Start (também conhecido como javaws, javaws ou JAWS ) é uma estrutura desenvolvida pela Sun Microsystems ( agora Oracle) que permite aos usuários iniciar o software aplicativo para a plataforma Java diretamente da Internet usando um navegador web. Alguns dos principais benefícios desta tecnologia incluem a versão perfeita de actualização para aplicações distribuídas globalmente e maior controle de alocação de memória para a máquina virtual Java [1].

- Banco de Dados: Oracle RAC $\rightarrow$ Em computação de banco de dados, o Oracle Real Application Clusters (RAC) - uma opção para o software de banco de dados Oracle produzido pela Oracle Corporation e introduziu em 2001 com Oracle9i - fornece software para clustering e alta disponibilidade em ambientes de banco de dados Oracle. Oracle Corporation inclui RAC com o Standard Edition, desde os nós estão agrupados usando o Oracle Clusterware [1].

- Framework de acesso ao banco de dados: Hibernate $\rightarrow$ O Hibernate é um framework para o mapeamento objeto-relacional escrito na linguagem Java, mas também é disponível em .Net como o nome NHibernate. Este framework facilita o mapeamento dos atributos entre uma base tradicional de dados relacionais e o modelo objeto de uma aplicação, mediante o uso de arquivos (XML) ou anotações Java [1]. 
- Serviço de mensageria: Oracle $\mathrm{OMQ} \rightarrow$ Serviço de mensageria utilizado para troca de mensagens.

- Servidores de aplicação virtualizados: VMWare $\rightarrow$ VMware é um software/máquina virtual que permite a instalação e utilização de um sistema operacional dentro de outro dando suporte real a software de outros sistemas operativos. Usando software de virtualização como o VMware é possível executar um ou mais sistemas operacionais simultaneamente num ambiente isolado, criando computadores completos (virtuais) a executar dentro de um computador físico que pode rodar um sistema operacional totalmente distinto. Do ponto de vista do utilizador e do software nem sequer se nota a diferença entre a máquina real e a virtual. É muito usado em centros de dados, pois permite criar redundância e segurança adicional sem recorrer a tantas máquinas físicas e distribuindo e aproveitando melhor os recursos das máquinas hospedeiras [1].

- Framework de logs: Logback $\rightarrow$ framework para geração de logs em sistemas Java.

- Comunicação cliente servidor baseada na troca de eventos assíncronos.

- Comunicação com nível 1: OPC UA $\rightarrow$ OPC Unified Architecture ( OPC UA) é um protocolo de comunicação M2M industrial para interoperabilidade desenvolvido pela Fundação OPC. É o sucessor para abrir plataforma de comunicações (OPC). Embora desenvolvido pela mesma organização, OPC UA difere significativamente de seu antecessor. O objetivo da Fundação para este projeto era fornecer um caminho para a frente do modelo de comunicações originais OPC (ou seja, o Microsoft Windows única bolsa processo COM / DCOM) para uma arquitetura orientada a serviços multiplataforma (SOA) para controle de processos, reforçando simultaneamente a segurança e fornecendo um modelo de informação. Após mais de três anos de trabalho de especificação e mais um ano para uma implementação do protótipo, a primeira versão da arquitetura unificada foi lançado em 2006 [1].

- Servidores OPC: ExaOPC e KepWare $\rightarrow$ Plataforma Aberta de Comunicações (OPC) é uma série de normas e especificações para telecomunicação industrial. Uma força-tarefa industrial indústria de automação desenvolvido o padrão original, em 1996, sob o nome de OLE for Process Control (Object Linking and Embedding for Process Control). OPC especifica a comunicação de dados da planta em tempo real entre dispositivos de controle de diferentes fabricantes. Após o lançamento inicial em 1996, a Fundação OPC foi criada para manter o padrão. [1] Como OPC foi adotado para além do campo de controle de processo, a Fundação OPC mudou o nome para abrir plataforma de comunicações em 2011. [2] A mudança em nome reflete as aplicações da tecnologia OPC para aplicações em automação predial, manufatura discreta, controle de processos e muitos outros. OPC também tem crescido para além seu original OLE (Object Linking and Embedding) implementação para incluir outras tecnologias de transporte de dados, incluindo o Microsoft .NET Framework, XML, e mesmo formato binário codificado TCP da Fundação OPC [1].

- Controle de código fontes: SVN $\rightarrow$ Apache Subversion (também conhecido por SVN) é um sistema de controle de versão desenhado especificamente para ser um substituto moderno do CVS, que se considera ter algumas limitações. O Subversion utiliza banco de dados Berkeley BD [1]. 
- Ferramenta de integração contínua: Jenkins $\rightarrow$ Jenkins é uma ferramenta de integração contínua de código aberto escrito em Java. O projeto foi bifurcado de Hudson depois de uma disputa com a Oracle. Jenkins fornece serviços de integração contínua para desenvolvimento de software. É um sistema em execução baseada em servidor em um servlet container como o Apache Tomcat. Ele suporta ferramentas de SCM, incluindo AccuRev, CVS, Subversion, Git, Mercurial, Perforce, Clearcase e RTC, e pode executar projetos baseados Apache Ant e Maven Apache, bem como scripts shell arbitrários e comandos de lote do Windows. O principal desenvolvedor de Jenkins é Kohsuke Kawaguchi. Compilações pode ser iniciado através de vários meios, incluindo a ser desencadeada por comprometer em um sistema de controle de versão, agendando através de um mecanismo cron -like, através da construção, quando outro constrói ter concluído, e solicitando uma Jbuild URL específica [1].

- Ferramenta de monitoramento: Zabbix $\rightarrow$ Zabbix é um software de monitoramento open source da empresa para as redes e aplicações, criadas por Alexei Vladishev. Ele é projetado para monitorar e acompanhar o status de vários serviços de rede, servidores e outro hardware de rede. Zabbix usa MySQL, PostgreSQL, SQLite, Oracle ou IBM DB2 para armazenar dados. O seu backend é escrito em $\mathrm{C}$ e a interface web é escrito em PHP. Zabbix oferece várias opções de monitoramento: verificações simples pode verificar a disponibilidade e capacidade de resposta dos serviços padrão, como SMTP ou HTTP sem instalar qualquer software na Estação monitorada, um agente Zabbix também pode ser instalado em UNIX e Windows hospeda para monitorar estatísticas como carga de CPU, utilização da rede , espaço em disco , etc, como uma alternativa para instalar um agente em hosts, Zabbix inclui suporte para monitoramento via SNMP , TCP e verifica ICMP, bem como sobre IPMI , JMX, SSH, Telnet e usando parâmetros personalizados . Zabbix suporta uma variedade de mecanismos de notificação quase em tempo real, incluindo XMPP [1].

\section{RESULTADOS E DISCUSSÃO}

Os novos sistemas dos Altos Fornos já estão implantados e em produção. Uma visão final de toda a arquitetura do sistema desenvolvido pode ser vista na figura abaixo: 
Z aut-app-zabbio01.amauto $\times$

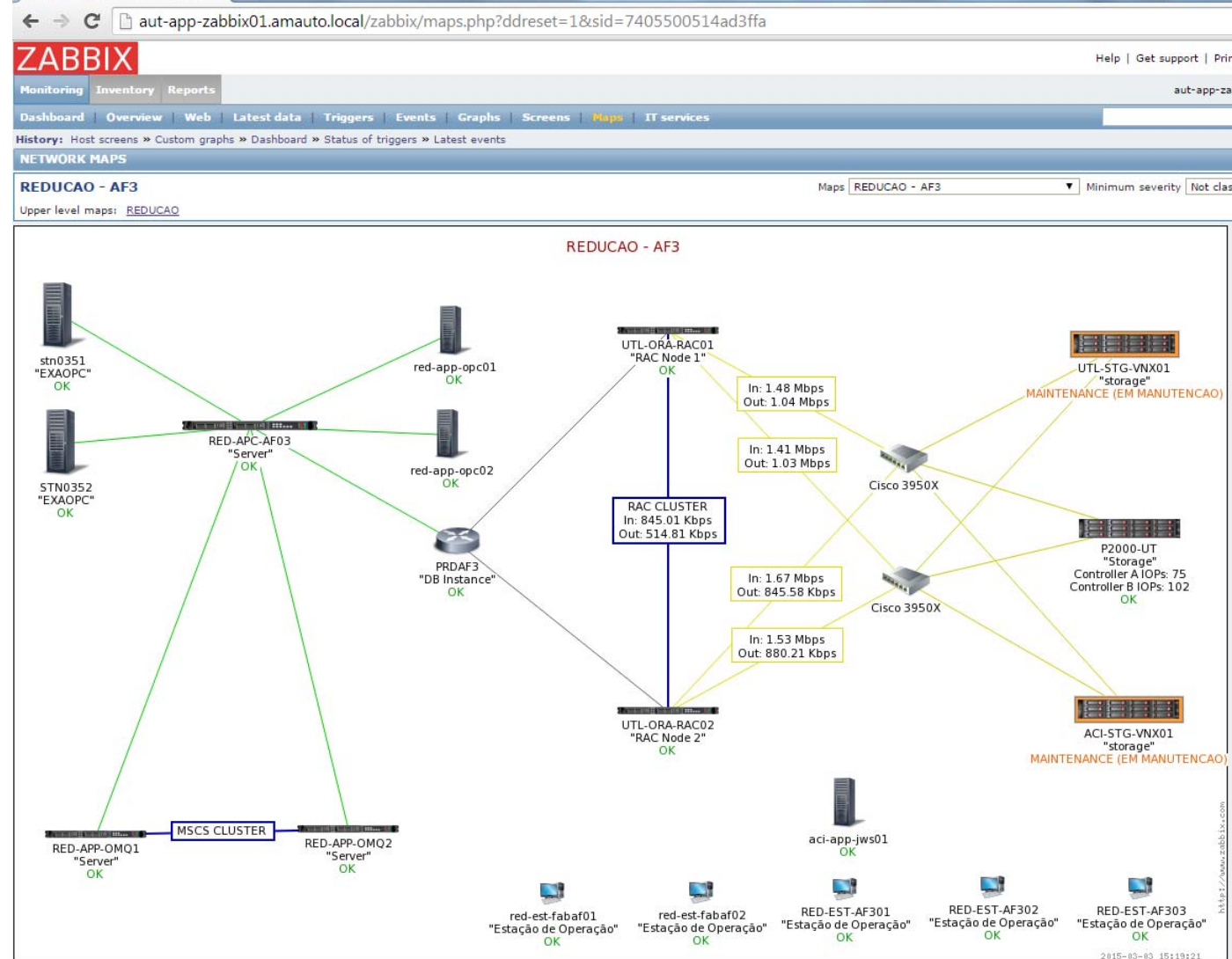

Figura 3. Visão Geral do Novo Sistema do Alto Forno 3

Abaixo estão listados todos os elementos da arquitetura do sistema do Alto Forno 3:

- STN0351 e STN0352: Servidores ExaOPC da Yokogawa.

- RED-APC-AF03: Servidor da aplicação do AF3.

- RED-APP-OMQ1 e 2: Servidor de mensageria utilizado para comunicação entre sistemas.

- RED-APP-OPC01 e 02: Servidor Kepware utilizado para comunicação com os ExaOPC.

- UTL-ORA-RAC01 e o2: Servidor de banco de dados Oracle RAC.

- RED-EST-FABXX: Estação de operação thin cliente.

Entre os principais benefícios dessa atualização tecnológica podemos listar:

- Facilidade de manutenção dos sistemas.

- Reuso das regras de negócio já criadas no Framework dos Altos Fornos.

- Alta disponibilidade da arquitetura com a utilização do VMWare HA.

- Automatização dos bakcups e restore dos servidores.

- Monitoração completa de todos os serviços e recursos utilizados pela aplicação.

Com relação aos benefícios financeiros obtidos com a adoção dessa estratégia podemos citar:

- Custo evitado em aquisição de licenças devido às centralizações de infraestrutura propostas no PDTA (Banco Centralizado e OMQ

- Ganhos de produtividade e de padronização no desenvolvimento dos sistemas fazendo reuso de uma base de software pré-construída que já endereça os principais elementos necessários para elaboração dos sistemas, 
como exemplo: Comunicação Nível 1 (OPC), Comunicação Nível 2/Nível 3, Classes de Armazenamento, Gestão de Paradas, Gestão de Turnos, Scheduling, tratativa de Alarmes/Eventos, Logging, Interface Gráfica (Telas)

- Podemos observar também uma redução de custo significativa ao longo da migração dos três sistemas:

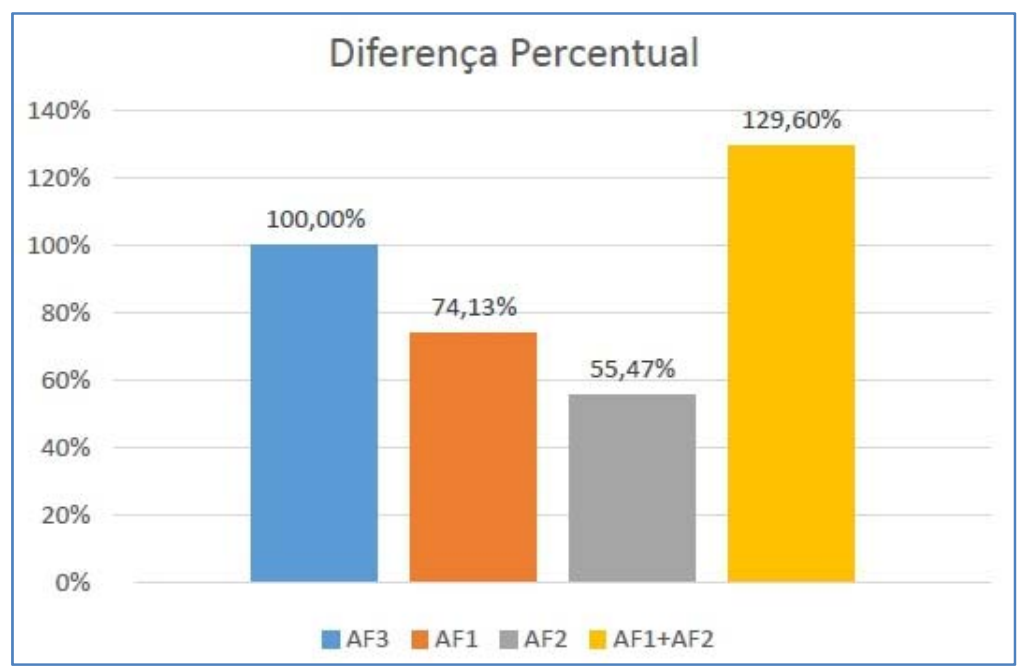

A tabela abaixo mostra um comparativo entre as telas do antigo e novo sistema:

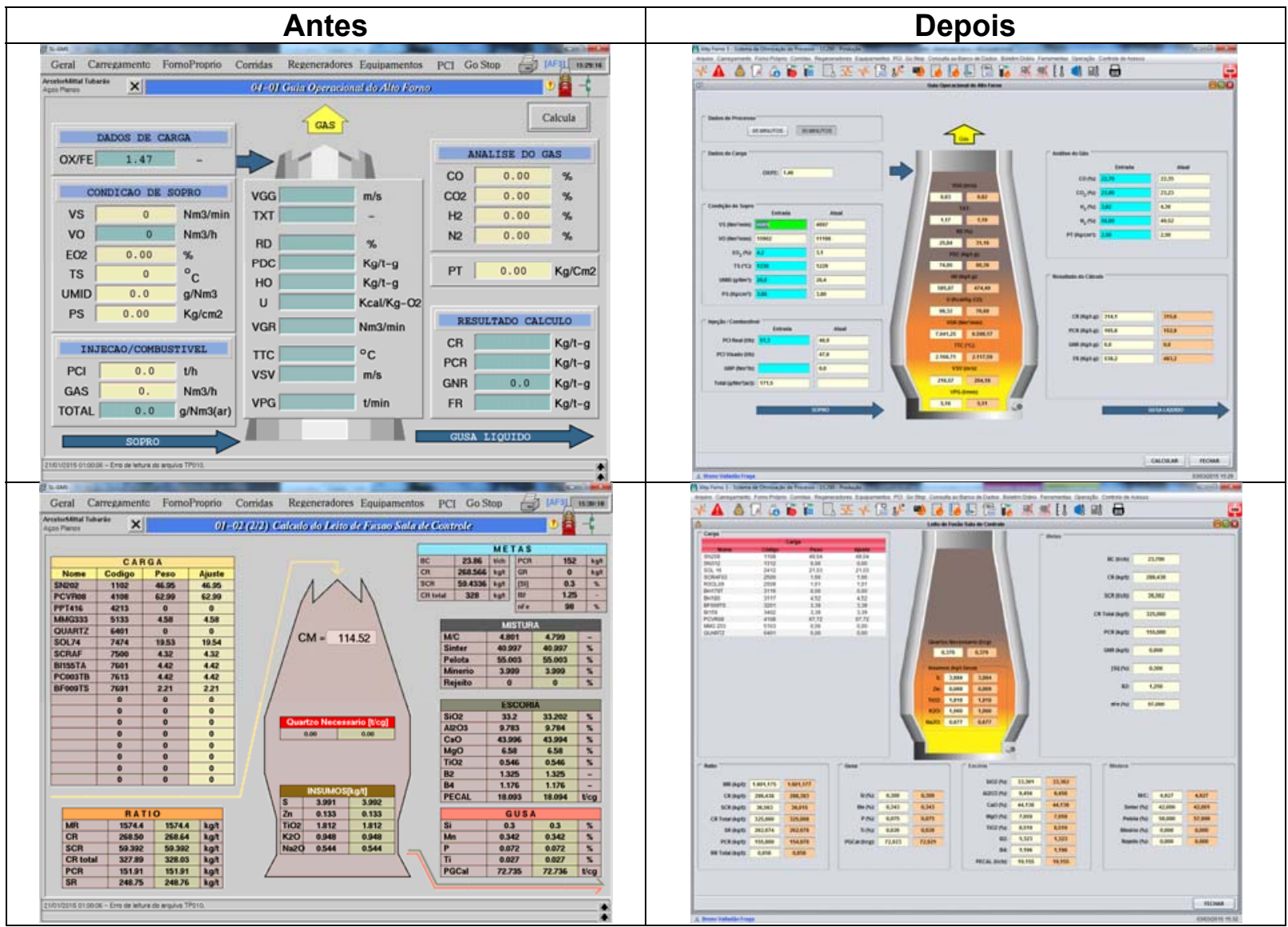

Tabela 1. Telas do novo sistema 


\section{CONCLUSÃO}

Há dois pontos distintos há analisar em todo este processo que estabelecem a conclusão.

O primeiro ponto está diretamente relacionado ao projeto dos Altos Fornos, onde foi possível, através da implantação, realizar o revamp do sistema do 3 altos fornos existentes, com um impacto quase nulo no processo, para uma tecnologia mais atualizada, que gera maiores condições de integração de novas funcionalidades e uniformizando a estrutura do sistema entre todos os equipamentos. Todo este processo permite à estrutura de automação um aumento de sua longevidade, bem como um incremento em sua estabilidade, motivo de preocupação principalmente devido a antiga estrutura de hardware (alphaservers).

O segundo ponto relaciona-se ainda com a uniformidade citada no parágrafo anterior. Esta uniformidade advém da adoção do Framework desenvolvido pela Automação no âmbito do seu Plano Diretor de Tecnologia, e o projeto dos Altos Fornos permitiu validar com muito sucesso uma premissa do projeto associada ao reaproveitamento de códigos. Apesar de todas as peculiaridades relativas aos diferentes equipamentos e estruturas de controle, ainda assim foi possível um grande aproveitamento de código fonte, trazendo impacto significativo tanto em relação aos custos do projeto, com também no seu prazo de conclusão. Diante dos atuais desafios da indústria do aço, esta situação passa a ser fundamental na busca tanto de produtividade da equipe própria de Automação, como também na agilidade de distribuição de melhorias com o impacto já em todos os equipamentos.

\section{REFERÊNCIAS}

1 Wkipedia. 